\title{
Hair Cortisol and Its Association With Psychological Risk Factors for Psychiatric Disorders: A Pilot Study in Adolescent Twins
}

\author{
Liz Rietschel, ${ }^{1, *}$ Fabian Streit, ${ }^{2, *}$ Gu Zhu, ${ }^{3}$ Kerrie McAloney, ${ }^{3}$ Clemens Kirschbaum, ${ }^{4}$ Josef Frank, ${ }^{2}$ \\ Narelle K. Hansell, ${ }^{3,5}$ Margaret J. Wright, ${ }^{3,5}$ John J. McGrath, ${ }^{5}$ Stephanie H. Witt, ${ }^{2}$ Marcella Rietschel, ${ }^{2}$ \\ and Nicholas G. Martin ${ }^{3}$ \\ ${ }^{1}$ Child and Adolescent Psychiatry, University Psychiatric Hospital, Bern, Switzerland \\ ${ }^{2}$ Department of Genetic Epidemiology in Psychiatry, Central Institute of Mental Health, Medical Faculty Mannheim, \\ University of Heidelberg, Mannheim, Germany \\ ${ }^{3}$ Genetic Epidemiology, Queensland Institute of Medical Research, Brisbane, Australia \\ ${ }^{4}$ Department of Psychology, Technische Universität Dresden, Dresden, Germany \\ ${ }^{5}$ Queensland Brain Institute and Centre for Advanced Imaging, University of Queensland, Brisbane, Australia
}

\begin{abstract}
Measuring cortisol in hair is a promising method to assess long-term alterations of the biological stress response system, and hair cortisol concentrations $(\mathrm{HCC})$ may be altered in psychiatric disorders and in subjects suffering from chronic stress. However, the pattern of associations between HCC, chronic stress and mental health require clarification. Our exploratory study: (1) assessed the association between HCC and perceived stress, symptoms of depression and neuroticism, and the trait extraversion (as a control variable); and (2) made use of the twin design to estimate the genetic and environmental covariance between the variables of interest. Hair samples from 109 (74 female) subjects (age range 12-21 years, mean 15.1) including 8 monozygotic (MZ) and 21 dizygotic (DZ) twin pairs were analyzed. Perceived stress was measured with the Perceived Stress Scale and/or the Daily Life and Stressors Scale, neuroticism, and extraversion with the NEO-Five Factor Inventory or the Junior Eysenck Personality Questionnaire, and depressive symptoms with the Somatic and Psychological Health Report. We found a modest positive association between HCC and the three risk factors - perceived stress, symptoms of depression, and neuroticism ( $r=0.22-0.33$ ) - but no correlation with extraversion (-0.06). A median split revealed that the associations between HCC and risk factors were stronger (0.47-0.60) in those subjects with HCC $>11.36$ $\mathrm{pg} / \mathrm{mg}$. Furthermore, our results suggest that the genetic effects underlying HCC are largely shared with those that influence perceived stress, depressive symptoms, and neuroticism. These results of our proof of principle study warrant replication in a bigger sample but raise the interesting question of the direction of causation between these variables.
\end{abstract}

Keywords: hair cortisol, stress, twin-study, depression, neuroticism, genetics

The adequate treatment of psychiatric disorders is burdened by their complex etiology. The joint action of multiple genetic and environmental factors leads to onset of illness, which usually starts with non-specific symptoms in adolescence (Lesch, 2004; Van Os et al., 2008). Thus, the identification of biological and psychological markers that underlie the development and progression of illness and link to relevant pathophysiology is of crucial importance to understanding the mechanisms leading to psychiatric disorders and hence to their prevention and treatment.

Chronic stress and an impaired ability to cope with it, both at the psychological and biological level, are thought to play a key role in the development and maintenance of psychiatric disorders (de Kloet et al., 2005). The most common biological marker to measure stress response is cortisol, which is released by the hypothalamic-pituitaryadrenal (HPA) axis. The HPA axis is the main endocrine mediator of the stress response, and it is modulated by and exerts feedback on brain regions involved in psychiatric

RECEIVED 20 April 2016; ACCEPTED 25 April 2016. First published online 4 July 2016.

ADDRESS FOR CORRESPONDENCE: Liz Rietschel, Child and Adolescent Psychiatry, University Psychiatric Hospital, Bern, Switzerland. E-mail: liz.rietschel@kjp.unibe.ch

* These authors contributed equally to this work. 
disorders; for example, the amygdala, the hippocampus, and the prefrontal cortex (Dedovic et al., 2009; de Kloet et al., 2005; Herman et al., 2003).

Studies in healthy population samples show an increase in cortisol in blood, urine, and saliva during and after exposure to short-term stressors and a decrease once the stressor disappears (Miller et al., 2007). The regulation of the HPA axis has been reported to be altered in patients suffering from depression, schizophrenia, and anxiety disorders, supporting the hypothesis of impaired ability to cope with stress in psychiatric disorders (Bradley \& Dinan, 2010; Faravelli et al., 2012; Herbert, 2013; Jones \& Moller, 2011). The measurement of cortisol in blood, saliva, or urine is a sensitive method to assess acute stress responses and reflects short-term cortisol release. However, single cross-sectional samples may not provide a valid measure of chronic stress and long-term alterations of HPA functioning as they are sensitive to daily circumstances such as circadian rhythm, nutrition, and so forth.

A relatively new method that enables the efficient assessment of cortisol release over a longer period is its measurement in hair of the scalp (Stalder et al., 2012). Scalp hair grows at an average rate of $1 \mathrm{~cm}$ per month, so cortisol assayed from a $3 \mathrm{~cm}$ hair segment closest to the scalp likely reflects the average release over the past 3 months and is therefore a potential biomarker of long-term altered HPA functioning. Initial studies showed altered hair cortisol concentrations (HCC) in unaffected adults with high levels of chronic stress and in cases suffering from psychiatric disorders (including bipolar disorders, depression, and anxiety disorder) compared with controls (reviewed in Herane Vives et al., 2015). More recent studies found similar associations in children and adolescents for objective stress and HCC (Rippe et al., 2016; Simmons et al., 2016; Vliegenthart et al., 2016).

These alterations in HCC may reflect causal mechanisms such as genetic determinants that jointly influence the level of HCC, and moderate affective symptoms and perceived stress (pleiotropy), or may be a direct consequence of the pathogenesis such that affective symptoms and perceived stress increase HCC; or both mechanisms may operate. However, few studies have assessed the association between HCC, affective symptoms, and perceived stress. Furthermore, to our knowledge, no study has assessed the association between HCC and the personality traits of neuroticism and extraversion, where the first is a risk factor for the development of psychiatric disorders and the latter is not (Kendler et al., 2006; Van Os \& Jones, 2001).

Many previous studies assessing the relationship between perceived stress and HCC found no significant or low correlations (Heinze et al., 2016; Staufenbiel et al., 2013; Streit et al., 2016). This so-called lack of psychoendocrine covariance, with endocrine response not coinciding with psychological stress, might be due to most studies assuming a linear association between hair cortisol and psychological mark- ers. It is possible though that a pathological threshold exists for cortisol as it does for other metabolite biomarkers such as creatinine, where only higher values are associated with renal damage and lower levels have no clinical implications (Singhal \& Saha, 2014).

The primary aim of our pilot study was to assess the association of HCC with psychological risk factors for psychiatric disorders - perceived stress, symptoms of depression, and neuroticism, and also the personality trait extraversion, for which there is no expected relation with cortisol. Our secondary aim was to explore the genetic and environmental causation of these associations by making use of the MZ and DZ twins in our sample.

\section{Materials and Methods}

\section{Sample}

Our sample comprised 135 adolescent or young adult twins (95 females) who had taken part in either the Brisbane Longitudinal Twin Study (BLTS; Wright \& Martin, 2004) or the Queensland Twin Imaging Study (QTIM study; Blokland et al., 2008) between 2009 and 2012. In the BLTS, psychiatric symptoms are assessed at ages 12,14 and 16 years (Hansell et al., 2012). The QTIMS is a follow-up study of the BLTS cohort, which involves investigation of twins aged 21-28 years. Zygosity was initially determined by a combination of standard questions (Martin \& Martin, 1975) and a photograph of the twin pairs, and subsequently confirmed by extensive genotyping. The sample consisted of 8 monozygotic (MZ) and 24 dizygotic (DZ) complete twin pairs, and 66 individual twins and 5 siblings. In both the $\mathrm{MZ}$ and DZ groups, female twin pairs were predominant.

Data of those subjects with a weight of $<7.5 \mathrm{mg}$ of hair cortisol were excluded ( $n=26$ which left $8 \mathrm{MZ}$ pairs, $21 \mathrm{DZ}$ pairs, and 51 unpaired twins or sibs for further analysis; for details see section below titled Hair Cortisol Analysis). The mean age of the sample was 15.1 years $(S D=1.99$; range $12-21$ years).

We are well aware that this is too small a sample for reliable heritability estimation, but our principal interest is in the relation between HCC and our psychological variables, and the genetic and environmental causes of any such correlation for which the power of analysis is greater given the multivariate nature of the data.

\section{Self-Report Measures}

For participants aged 12,14, and 16 years, perceived stress in the past month was measured using the 30-item Daily Life and Stressors Scale (DLSS; Kearney et al., 1993). The trait variables neuroticism and extraversion were measured using the Junior Eysenck Personality Questionnaire (JEPQ; Eysenck, 1972) with 20 items for neuroticism and 24 items for extraversion. For participants aged 16 years or older, perceived stress was measured using the 10-item Perceived Stress Scale (PSS; Cohen et al., 1983), and neuroticism and 
extraversion were measured using the NEO-Five Factor Inventory revised version (Neo-FFI-R; McCrae \& Costa Jr., 2004), each with 12 items for neuroticism and extraversion. Depressive symptoms at all ages were assessed using the 34item Somatic and Psychological Health Report (SPHERE; Hansell et al., 2012; Hickie et al., 2001).

\section{Hair Cortisol Analysis}

Sample collection and preparation were performed as described elsewhere (Kirschbaum et al., 2009). In brief, a swatch of hair about $3 \mathrm{~mm}$ in cross-section from the back of the head (posterior vertex position) was cut with fine scissors as close as possible to the scalp. As hair grows at an average speed of $1 \mathrm{~cm}$ per month, the $3-\mathrm{cm}$ segment proximal to the scalp can be assumed to reflect cortisol secretion during the preceding 3 months (LeBeau et al., 2011), and our sample was trimmed from the distal end to this length. Hair washing and steroid extraction were performed as described in Davenport et al. (2006). The hair samples were then micro-centrifuged at 10,000 rpm for $2 \mathrm{~min}$. A $1 \mathrm{ml}$ volume of the clear supernatant was then transferred into a new cryo vial in order to allow the alcohol to evaporate at $60{ }^{\circ} \mathrm{C}$ under a constant stream of nitrogen until the samples were completely dry. Finally, $0.4 \mathrm{ml}$ of phosphate buffer was added and the tube was vortexed for $15 \mathrm{~s}$ before cortisol was assayed using a commercial immunoassay kit as described by Kirschbaum et al. (2009).

Ideally, the HCC assays should be performed on a hair sample weighing at least $10 \mathrm{mg}$. However, for some individuals ( $n=46$, mainly males) this amount of hair had not been collected. Preliminary analysis of the entire sample of observations revealed a strong negative correlation $(r=-$ 0.49 ) between the weight of the hair sample being analyzed and the HCC. We have no explanation for this effect, which was observed in men and women and both did not differ in average cortisol levels $\left(t_{133}=0.24, p=.81\right)$. On inspection, this relation appeared to apply only to the smallest samples and to asymptote above a sample weight of $7.5 \mathrm{mg}$ so we used this as a lower limit for inclusion in the study.

\section{Data Analysis}

Due to skewness, hair cortisol concentration ( mean $=14.07$ $\mathrm{pg} / \mathrm{mg}, S D=6.48$, range $1.61-41.33$ ) was log transformed for all analyses.

To compare neuroticism scores between participants, the neuroticism, and extraversion sum-scores of the NEO-FFI and the JEPQ-scores were separately $z$-transformed and then combined. For the assessment of perceived stress measured by the PSS (Cohen et al., 1983) and the DLSS (Kearney et al., 1993), and depressive symptoms measured by the SPHERE (Hickie et al., 2001), item response theory (IRT) analyses were performed (Wray et al., 2008) to harmonize data. The details and advantages of IRT analysis are described elsewhere (Rietschel et al., 2014).

\section{TABLE 1}

Correlations (95\% C I) Between Psychological Variables and HCC in the Whole Sample $(n=109)$, and after Median Split (HCC $>\backslash \leq 11.36 \mathrm{pg} / \mathrm{mg}$ )

\begin{tabular}{lccl}
\hline & $\begin{array}{l}\text { Whole sample } \\
(n=109)\end{array}$ & $\begin{array}{l}\text { High cortisol } \\
(n=53)\end{array}$ & $\begin{array}{l}\text { Low cortisol } \\
(n=56)\end{array}$ \\
\hline Stress & $0.22(0.03,0.41)$ & $0.47(0.23,0.78)$ & $0.09(-0.19,0.36)$ \\
Depression & $0.33(0.15,0.53)$ & $0.60(0.41,0.97)$ & $0.03(-0.24,0.30)$ \\
Neuroticism & $0.28(0.10,0.48)$ & $0.49(0.26,0.81)$ & $0.15(-0.12,0.42)$ \\
Extraversion & $-0.06(-0.13,0.25)$ & $-0.07(-0.21,0.35)$ & $0.12(-0.15,0.39)$ \\
\hline
\end{tabular}

Preliminary analysis of the association between hair cortisol and the different psychological measures (e.g., ratings of perceived stress and neuroticism) was complicated by the fact that our twin study contained two children from the same family, leading to non-independent observations. As such, we randomly split the data so that no family member would be in the same group; Spearman correlations were then performed to examine the relationship between neuroticism, extraversion, symptoms of depression, perceived stress, and hair cortisol, using pairwise deletion of missing data. Afterwards, correlations were meta-analytically combined using Hedges method, which adjusts estimated standard errors and sample size. To distinguish subjects with high versus low hair cortisol, a median split was carried out.

\section{Model Fitting}

To allow use of data from all individual twins, including those without co-twins, and from participants with missing outcome measures, structural equation models (SEM) were fitted using the full information maximum likelihood (FIML) method implemented in Mx (Neale et al., 2004). Details of the twin design and analytical methods, including assumption testing and multivariate modeling (below), are described elsewhere (Neale \& Cardon, 1992). For multivariate analysis, a Cholesky decomposition for the ACE model was compared with Cholesky decompositions for simpler AE, CE, and E models. Despite evidence for a nonlinear relationship between HCC and the affect variables, we carried out the variance components analysis, assuming only linear relations given our small sample size. The fit of each model was assessed by the differences in log likelihood between the sub and the full models. For all models, sex and age were fitted as fixed effects.

\section{Results}

\section{Relation Between HCC and Our Affect Measures}

Preliminary inspection of scatterplots of HCC with behavioral measures suggested non-linear relationships. We therefore performed a median split at $\mathrm{HCC}=11.36 \mathrm{pg} / \mathrm{mg}$ and estimated correlations for the high and low halves of the sample as well as the whole. Table 1 reveals the following: (a) correlations between HCC and the three risk factors 
TABLE 2

Pearson Correlations Between All Variables in DZ (21 Pairs) and MZ (8 Pairs) Twins (MZ in lower, DZ in Upper Triangle) Corrected for Age and Sex

\begin{tabular}{|c|c|c|c|c|c|c|c|c|c|c|c|}
\hline & & \multicolumn{5}{|c|}{ Twin 1} & \multicolumn{5}{|c|}{ Twin 2} \\
\hline & & Stress & Depression & Neuroticism & Extraversion & Hair cortisol & Stress & Depression & Neuroticism & Extraversion & Hair cortisol \\
\hline & Stress & 1 & 0.53 & 0.66 & -0.26 & 0.19 & 0.24 & -0.04 & 0.64 & 0.05 & -0.63 \\
\hline & Depression & 0.68 & 1 & 0.46 & -0.09 & 0.36 & 0.35 & 0.05 & 0.11 & -0.03 & 0.21 \\
\hline & Neuroticism & 0.84 & 0.81 & 1 & -0.10 & -0.10 & 0.25 & -0.05 & 0.04 & 0.09 & -0.01 \\
\hline \multirow[t]{2}{*}{ Twin 1} & Extraversion & 0.23 & 0.01 & 0.48 & 1 & -0.09 & -0.23 & -0.34 & -0.31 & -0.15 & 0.19 \\
\hline & Hair cortisol & 0.54 & 0.87 & 0.72 & 0.31 & 1 & -0.10 & 0.17 & -0.09 & 0.09 & 0.63 \\
\hline \multirow[t]{5}{*}{ Twin 2} & Stress & 0.61 & 0.74 & 0.71 & -0.04 & 0.52 & 1 & 0.73 & 0.69 & -0.08 & 0.28 \\
\hline & Depression & 0.72 & 0.73 & 0.86 & 0.17 & 0.56 & 0.90 & 1 & 0.76 & -0.15 & 0.29 \\
\hline & Neuroticism & 0.53 & 0.68 & 0.75 & 0.05 & 0.46 & 0.80 & 0.91 & 1 & -0.18 & 0.31 \\
\hline & Extraversion & 0.40 & 0.35 & 0.32 & 0.08 & 0.45 & 0.48 & 0.66 & 0.30 & 1 & -0.08 \\
\hline & Hair cortisol & 0.15 & 00.09 & 0.25 & 0.65 & 0.20 & 0.15 & 0.10 & 0.13 & -0.42 & 1 \\
\hline
\end{tabular}


— perceived stress, symptoms of depression, and neuroticism - are modest and significant in the whole sample but high and significant for the group of high HCC (HCC $>11.36 \mathrm{pg} / \mathrm{mg}$ ); (b) there are no significant associations between HCC and the three risk factors in the low-cortisol group ( $\mathrm{HCC} \leq 11.36 \mathrm{pg} / \mathrm{mg}$ ); (c) there is no significant association between extraversion and HCC.

\section{Twin Correlations}

The twin- correlations between the psychological variables of perceived stress, neuroticism, extraversion, and HCC are displayed in Table 2. As expected, correlations between MZ twins are higher than for DZ twins for neuroticism, perceived stress, and extraversion. However, unexpectedly, the correlation for HCC is higher in the DZ $(r=0.63)$ than in the MZ $(r=0.20)$ group, though not significantly so, given these small sample sizes.

\section{Genetic Covariance Between HCC and Affect Variables}

Rather than focus on the zero estimate of heritability for HCC (which is likely a stochastic result due to low numbers), we are more interested in evidence for genetic covariance between HCC and the psychological variables. To investigate this, we fitted a series of Cholesky decomposition models, including variously $\mathrm{A}, \mathrm{C}$, and $\mathrm{E}$, as sources of covariation. The aim of the analyses was to determine whether genetic influences specific to HCC exist after perceived stress, neuroticism, and depressive symptoms have been accounted for. Therefore, perceived stress was used as the first, depressive symptoms as the second, neuroticism as the third, and HCC as the fourth latent factor. We began by fitting an ACE Cholesky model, but no significant worsening of the fit was observed when either the $\mathrm{C}$ matrix was fixed to zero $\left(\chi^{2}{ }_{10}=4.51\right)$ or the $\mathrm{A}$ matrix was fixed to zero $\left(\chi^{2}{ }_{10}=4.07\right)$. In fact, both $\mathrm{A}$ and $\mathrm{C}$ matrices could be fixed to zero without fatally affecting fit $\left(\chi^{2}{ }_{20}=\right.$ 18.22) underlining the fundamental lack of power of our experiment. Nevertheless, the point estimates from the ACE Cholesky decomposition are of great interest. Table 3 shows that the estimates of factor loadings for the A matrix are substantial, those for the $\mathrm{C}$ matrix are negligible except for $\mathrm{C}$ $(4,4)$, the specific loading on HCC, reflecting the low $r_{\mathrm{mz}}$ for HCC noted above. Indeed, even the cross-loadings for the E matrix are considerably smaller than their corresponding elements in the A matrix. Notwithstanding the deficiencies in power of our study, our results point strongly to the predominant importance of genetic factors in shaping the correlations observed between HCC and the psychological variables.

Nevertheless, because A and C estimates tend to be intimately confounded in the twin model, and because the point estimates from Figure 1 suggest that the dominant source by far is A, for simplicity, in Figure 1 we show the standardized path coefficients for the AE Cholesky decomposition (these must be squared to obtain variance compo-

\section{TABLE 3}

Cholesky Decomposition for ACE Model

\begin{tabular}{lllll}
\hline A & 1 & 2 & 3 & 4 \\
\hline Stress & 0.77 & & & \\
Depression & 0.57 & 0.60 & & \\
Neuroticism & 0.43 & 0.49 & 0.48 & 0.26 \\
Hair cortisol & 0.37 & 0.37 & 0.34 & \\
C & & & & \\
Stress & 0.20 & & & \\
Depression & 0.04 & 0.02 & 0.09 & \\
Neuroticism & 0.11 & 0.02 & 0.18 & \\
Hair cortisol & 0.21 & 0.05 & & \\
E & & & & \\
Stress & 0.61 & & 0.40 \\
Depression & 0.28 & 0.49 & 0.41 & \\
Neuroticism & 0.31 & 0.25 & 0.17 & \\
Hair cortisol & 0.19 & 0.08 & \\
\hline Note: Latent factor loadings are standardized to unit variance and must be \\
\multicolumn{4}{r}{ squared to obtain standardized variance components. }
\end{tabular}

nents), remembering that, to a small extent, the A estimates are confounded with some shared environmental effects. Under this simplified model, genetic factors would account for more than half the total variance for all four variables. Further, the first genetic factor (A1), which loads primarily on perceived stress and accounts for $63.3 \%$ of its variance, also accounts for $32.2 \%$ of the variance in depressive symptoms, $20 \%$ of the variance for neuroticism, and $11.4 \%$ of the variance for HC. The second genetic factor (A2), which loads primarily on depressive symptoms $(35.7 \%)$, also accounts for $24.2 \%$ of the variance in neuroticism and $15.4 \%$ in HCC. The third genetic factor (A3), which loads primarily on neuroticism (23.2\%), also accounts for $11.6 \%$ of the variance in $\mathrm{HC}$. This leaves a specific genetic contribution (A4) to HCC that accounts for $24.9 \%$ of its variance. Decomposition of the non-shared environmental covariance shows that the first factor (E1) accounts for $36.7 \%$ of the variance for neuroticism and also accounts for $7.6 \%$ of the variance in depressive symptoms, $9.6 \%$ of the variance in perceived stress, and $4 \%$ of the variance for $\mathrm{HC}$. The second factor (E2), which loads on depressive symptoms (24.6\%), also accounts for $6 \%$ of the variance in perceived stress and $0.3 \%$ of the variance for HCC. The third factor (E3) loads on neuroticism and accounts for $17 \%$ of its variance and $3 \%$ on HCC. The specific non-shared environmental contribution to HCC (E4) accounts for $29.9 \%$ of its variance.

\section{Genetic Correlation Between HCC and Affect Variables}

Also for simplicity, the genetic and environmental correlations between the four variables, derived from the $\mathrm{AE}$ Cholesky analysis, are shown in Table 4.

We can see that the correlations between the three psychological variables largely derive from genetic sources. The correlations between these three and HCC are also much more strongly genetically than environmentally influenced. 
TABLE 4

Additive Genetic and Unshared Environmental Correlations Between Psychological Variables and HCC Corrected for Age and Sex

\begin{tabular}{llllll}
\hline & & \multicolumn{4}{c}{ Environmental } \\
\cline { 3 - 6 } & & Stress & Depression & Neuroticism & Hair cortisol \\
\hline \multirow{2}{*}{ Genetic } & Stress & & 0.32 & 0.56 & 0.15 \\
& Depression & 0.86 & - & 0.34 & 0.01 \\
& Neuroticism & 0.77 & 0.98 & - & 0.10 \\
& Hair cortisol & 0.37 & 0.53 & 0.43 & - \\
\hline
\end{tabular}

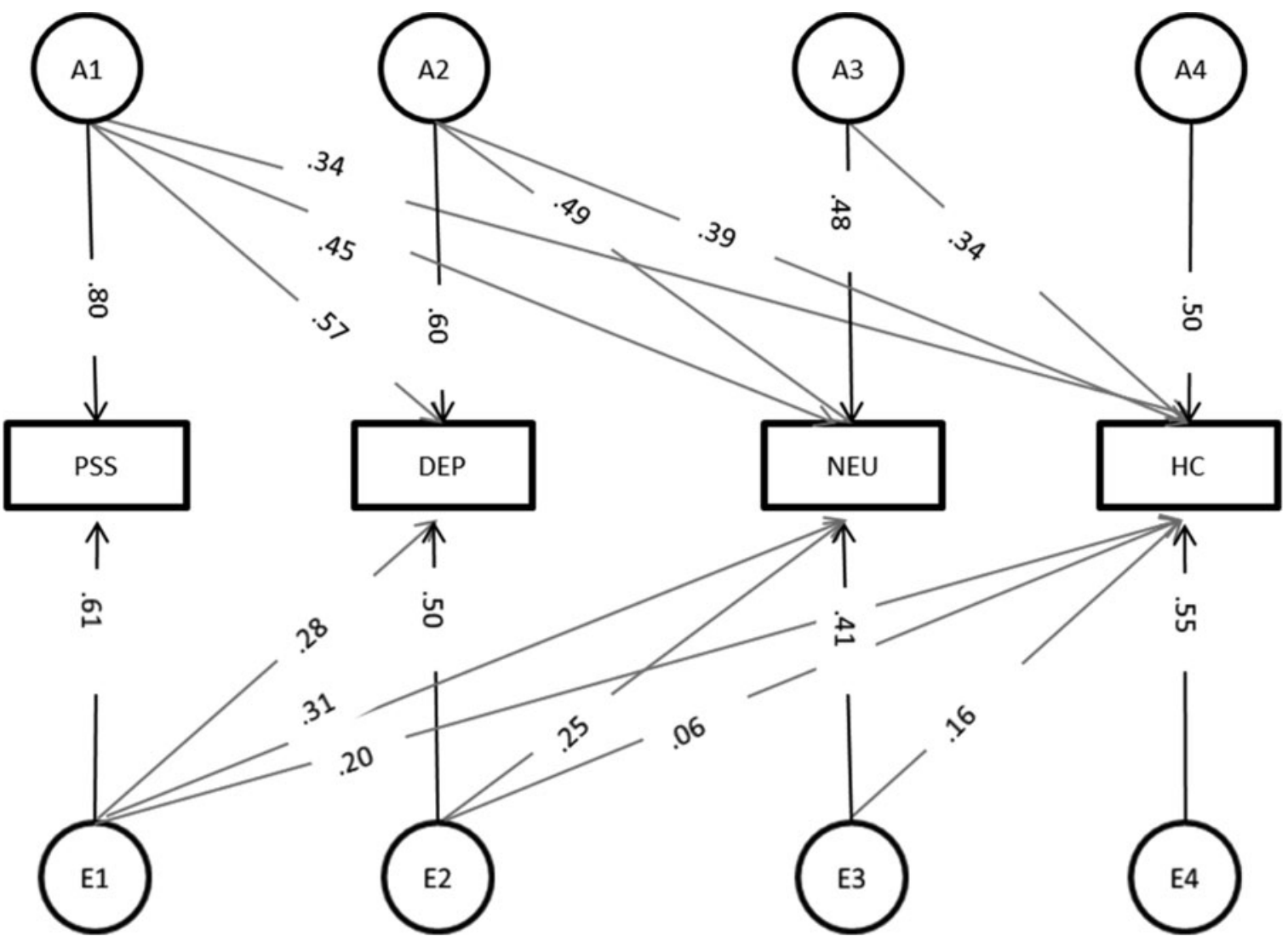

\section{FIGURE 1}

Cholesky decomposition for AE model. Latent factor loadings are standardized to unit variance and must be squared to obtain standardized variance components. A1-A4 additive genetic factors, E1-E4 unique environmental factors.

\section{Discussion}

The aim of this exploratory study was to assess the association between HCC and three affect variables we might reasonably expect to be associated with it: perceived stress, symptoms of depression and neuroticism. We also included extraversion as a control variable with no a priori expectation of association. Our sample included a small number of complete $\mathrm{MZ}$ and $\mathrm{DZ}$ twin pairs, and these enabled us to explore the genetic and environmental contributions to covariation between our variables. In particular, we were interested in the causes of covariation between HCC and the affect variables. Finally, we were interested in whether relationships between HCC and the affect variables are lin- ear, and we found some evidence that the relationship is much stronger in those with high HCC, suggesting there might be some threshold effect.

Our results show moderate but significant associations between HCC and neuroticism, perceived stress, and depressive symptoms, respectively $(r=0.22-0.33)$. Interestingly, splitting the sample into low versus high HCC revealed that lower levels of HCC seem to be unrelated to these psychological variables whereas the associations in the high-HCC group are substantial and significant $(r=$ 0.47-0.60). Furthermore, our study suggests strongly that these associations are genetic in origin. As expected, however, extraversion is not associated with HCC in either the 
high- or the low-HCC group, and this accords with previous studies of other cortisol markers (Munafò, 2006; Wilson et al., 2015).

Positive correlations between perceived stress and cortisol markers have been reported previously, even though the reports are not consistent (Staufenbiel et al., 2013; Vanaelst et al., 2012). These inconsistencies, together with our finding that the association is stronger in the high-HCC group, might indicate a potential pathological threshold for HCC; although, mindful of our small sample size, we have to regard this finding most tentatively, and clearly it needs to be replicated in a much larger dataset. To our knowledge, no previous study has suggested the existence of such a threshold, although others have suggested a more complex non-linear relationship (Wells et al., 2014).

In monkeys, the heritability for HCC has been estimated at 30\% (Fairbanks et al., 2011), while other twin studies in humans suggest that genetic factors account for $62 \%$ of the variance of saliva and serum cortisol (Bartels et al., 2003). Due to the small sample size, we were not able to reliably assess the heritability of HCC; the DZ correlation for HCC is high $(r=0.63)$ and most likely the very low MZ correlation ( $r=0.20)$ reflects our having only eight MZ twin pairs; we are currently collecting a much larger sample that will give us a more reliable estimate.

Most pertinent to the main aim of our study, our results suggest that the association between HCC and the affect variables seems to be largely driven by genetic factors. This supports the hypothesis of a common genetic association between all four measures, whereas environmental factors do not seem to contribute much to covariation. This is consistent with our results from a much larger twin sample (of which the present study uses a subsample) in which we estimated high genetic covariances between neuroticism, depression, and perceived stress (Rietschel et al., 2014).

The most obvious limitation of our study is the small sample size; this will be rectified in our forthcoming study with a sample six times larger than here. The much greater limitation, however, is that even if the relationships we observe here are replicated with increased significance, it will not elucidate the direction of causation in the correlation between these variables. While it is possible that perceived stress is environmentally induced and in turn induces high HCC, the reverse could also be true. Another suggestion from our present results though is compatible with a pleiotropic explanation, in which the same genes simultaneously influence both HCC and the psychological variables. Elucidation of this conundrum might be advanced by sensitive longitudinal studies, or as a byproduct of large-scale GWAS studies that enable construction of powerful instrumental variables for use in Mendelian randomization analyses (Richmond et al., 2016). Larger samples of MZ twins with varying degrees of discordance for HCC and the other measures here may also shed light on the nature and direction of causation between these variables.

In conclusion, our pilot study provides new evidence that HCC is related to psychological variables that elevate the risk for psychiatric disorders but not extraversion. Further, these correlations are seen most strongly in the higher range of HCC values, suggesting some sort of threshold effect. Most interestingly, analysis of these associations in $\mathrm{MZ}$ and DZ twins suggest that they are largely driven by genetic rather than either familial or idiosyncratic environmental influences. However, the direction of causation between these variables remains unclear.

\section{Acknowledgments}

We thank twins and their family members who participated in the BLTS and provided hair and blood samples, as well as nurses Natalie Garden and Reshika Chand, who made categorical ratings of hair curliness; and we thank Kerrie McAloney for coordination.

\section{Financial Support}

Funding for collection of hair samples from twins was provided by Australian NHMRC grants to NGM. Funding for measurement of hair samples (by Mark Brims, BSC Electronics, Perth) and for salary for Yvonne Ho was provided by an Australian Research Council Linkage Grant (LP 110100121: P.I. Dennis McNevin, University of Canberra) with contributing funding from Identitas Inc (principals Mike Fitzgerald (Monaco), Laurence Rubin (Toronto) via the Visigen Consortium convened by Tim Spector (Kings College London); further contributions were from the Victorian Police Forensic Services Department (Runa Daniels, Roland van Oorschot), the Australian Federal Police Forensics (Simon Walsh, Paul Kirkbride), and Unisys Australia (David Chadwick). Hair cortisol assays were funded by a grant to MR from the German Federal Ministry of Education and Research (BMBF) through the Integrated Network IntegraMent (Integrated Understanding of Causes and Mechanisms in Mental Disorders, grant BMBF01ZX1314G), under the auspices of the e:Med Programme.

\section{Conflicts of Interest}

None.

\section{Ethical Standards}

The authors assert that all procedures contributing to this work comply with ethical standards of the relevant national and institutional committees on human experimentation and with the Helsinki Declaration of 1975, as revised in 2008. The project was approved by the QIMR Human Research Ethics Committee. 


\section{References}

Bartels, M., Van den Berg, M., Sluyter, F., Boomsma, D. I., \& de Geus, E. J. C. (2003). Heritability of cortisol levels: Review and simultaneous analysis of twin studies. Psychoneuroendocrinology, 28, 121-137.

Blokland, G. A., McMahon, K. L., Hoffman, J., Zhu, G., Meredith, M., Martin, N. G., ... Wright, M. J. (2008). Quantifying the heritability of task-related brain activation and performance during the N-back working memory task: A twin fMRI study. Biological Psychology, 79, 70-79.

Bradley, A. J., \& Dinan, T. G. (2010). A systematic review of hypothalamic-pituitary-adrenal axis function in schizophrenia: Implications for mortality. Journal of Psychopharmacology, 24, 91-118.

Cohen, S., Kamarck, T., \& Mermelstein, R. (1983). A global measure of perceived stress. Journal of Health and Social Behavior, 24, 385-396.

Davenport, M. D., Tiefenbacher, S., Lutz, C. K., Novak, M. A., \& Meyer, J. S. (2006). Analysis of endogenous cortisol concentrations in the hair of rhesus macaques. General and Comparative Endocrinology, 147, 255-261.

Dedovic, K., Duchesne, A., Andrews, J., Engert, V., \& Pruessner, J. C. (2009). The brain and the stress axis: The neural correlates of cortisol regulation in response to stress. Neuroimage, 47, 864-871.

de Kloet, E. R., Joels, M., \& Holsboer, F. (2005). Stress and the brain: From adaptation to disease. Nature Reviews Neuroscience, 6, 463-475.

Eysenck, S. G. (1972). Junior eysenck personality inventory. San Diego, CA: Educational and Industrial Testing Service (EdITS).

Fairbanks, L. A., Jorgensen, M. J., Bailey, J. N., Breidenthal, S. E., Grzywa, R., \& Laudenslager, M. L. (2011). Heritability and genetic correlation of hair cortisol in vervet monkeys in low and higher stress environments. Psychoneuroendocrinology, 36, 1201-1208.

Faravelli, C., Lo Sauro, C., Lelli, L., Pietrini, F., Lazzeretti, L., Godini, L., \& Ricca, V. (2012). The role of life events and HPA axis in anxiety disorders: A review. Current Pharmaceutical Design, 18, 5663-5674.

Hansell, N. K., Wright, M. J., Medland, S. E., Davenport, T. A., Wray, N. R., Martin, N. G., \& Hickie, I. B. (2012). Genetic co-morbidity between neuroticism, anxiety/depression and somatic distress in a population sample of adolescent and young adult twins. Psychological Medicine, 42, 1249-1260.

Heinze, K., Lin, A., Reniers, R. L. E. P., \& Wood, S. J. (2016). Longer-term increased cortisol levels in young people with mental health problems. Psychiatry Research, 236, 98-104.

Herane Vives, A., De Angel, V., Papadopoulos, A., Strawbridge, R., Wise, T., Young, A. H., \& Cleare, A. J. (2015). The relationship between cortisol, stress and psychiatric illness: New insights using hair analysis. Journal of Psychiatric Research, $70,38-49$.

Herbert, J. (2013). Cortisol and depression: Three questions for psychiatry. Psychological Medicine, 43, 449-469.

Herman, J. P., Figueiredo, H., Mueller, N. K., Ulrich-Lai, Y., Ostrander, M. M., Choi, D. C., \& Cullinan, W. E. (2003).
Central mechanisms of stress integration: Hierarchical circuitry controlling hypothalamo-pituitary-adrenocortical responsiveness. Frontiers in Neuroendocrinology, 24, 151180.

Hickie, I. B., Davenport, T. A., Hadzi-Pavlovic, D., Koschera, A., Naismith, S. L., Scott, E. M., \& Wilhelm, K. A. (2001). Development of a simple screening tool for common mental disorders in general practice. The Medical Journal of Australia, 175, S10-17.

Jones, T., \& Moller, M. D. (2011). Implications of hypothalamic-pituitary-adrenal axis functioning in posttraumatic stress disorder. Journal of the American Psychiatric Nurses Association, 17, 393-403.

Kearney, C. A., Drabman, R. S., \& Beasley, J. F. (1993). The trials of childhood: The development, reliability, and validity of the daily life stressors scale. Journal of Child and Family Studies, 2, 371-388.

Kendler, K. S., Gatz, M., Gardner, C. O., \& Pedersen, N. L. (2006). Personality and major depression: A Swedish longitudinal, population-based twin study. Archives of General Psychiatry, 63, 1113-1120.

Kirschbaum, C., Tietze, A., Skoluda, N., \& Dettenborn, L. (2009). Hair as a retrospective calendar of cortisol production-Increased cortisol incorporation into hair in the third trimester of pregnancy. Psychoneuroendocrinology, 34, 32-37.

LeBeau, M. A., Montgomery, M. A., \& Brewer, J. D. (2011). The role of variations in growth rate and sample collection on interpreting results of segmental analyses of hair. Forensic Science International, 210, 110-116.

Lesch, K. P. (2004). Gene environment interaction and the genetics of depression. Journal of Psychiatry \& Neuroscience, 29(3), 174-184.

Martin, N. G., \& Martin, P. G. (1975). The inheritance of scholastric abilities in a sample of twins. I. Ascertainments of the sample and diagnosis of zygosity. Annals of Human Genetics, 39, 213-218.

McCrae, R. R., \& Costa Jr, P. T. (2004). A contemplated revision of the NEO Five-Factor Inventory. Personality and Individual Differences, 36, 587-596.

Miller, G. E., Chen, E., \& Zhou, E. S. (2007). If it goes up, must it come down? Chronic stress and the hypothalamicpituitary-adrenocortical axis in humans. Psychological Bulletin, 133, 25-45.

Munafò, M. R., Lee, L., Ayres, R., Flint, J., Goodwin, G., \& Harmer, C. J. (2006). Early morning salivary cortisol is not associated with extraversion. Personality and Individual Differences, 40(2), 395-400.

Neale, M. C., Boker, S. M., Xie, G., \& Maes, H. H. (2004). Mx: statistical modeling (6th ed.,). Richmond, VA: Department of Psychiatry, Virginia Commonwealth University.

Neale, M. C., \& Cardon, L. R. (1992). Methodology for genetic studies of twins and families. Dordrecht, Netherlands: Springer.

Richmond, R. C., Sharp, G. C., Ward, M. E., Fraser, A., Lyttleton, O., McArdle, W. L., \& Relton, C. L. (2016). DNA methylation and body mass index: Investigating identified 
methylation sites at HIF3A in a causal framework. Diabetes, 65, 1231-1244.

Rietschel, L., Zhu, G., Kirschbaum, C., Strohmaier, J., Wüst, S., Rietschel, M., \& Martin, N. G. (2014). Perceived stress has genetic influences distinct from neuroticism and depression. Behavior Genetics, 44, 639-645.

Rippe, R. C. A., Noppe, G., Windhorst, D. A., Tiemeier, H., van Rossum, E. F. C., Jaddoe, V. W. V., ... van den Akker, E. L. T. (2016). Splitting hair for cortisol? Associations of socio-economic status, ethnicity, hair color, gender and other child characteristics with hair cortisol and cortisone. Psychoneuroendocrinology, 66, 56-64.

Simmons, J. G., Badcock, P. B., Whittle, S. L., Byrne, M. L., Mundy, L., Patton, G. C., ... Allen, N. B. (2016). The lifetime experience of traumatic events is associated with hair cortisol concentrations in community-based children. Psychoneuroendocrinology, 63, 276-281.

Singhal, N., \& Saha, A. (2014). Bedside biomarkers in pediatric cardio renal injuries in emergency. International Journal of Critical Illness and Injury Science, 4, 238-246.

Streit, F., Memic, A., Hasandedić, L., Rietschel, L., Frank, J., Lang, M., Rietschel, M. (2016). Perceived stress and hair cortisol: Differences in bipolar disorder and schizophrenia. Psychoneuroendocrinology, 69, 26-34.

Stalder, T., Steudte, S., Miller, R., Skoluda, N., Dettenborn, L., \& Kirschbaum, C. (2012). Intraindividual stability of hair cortisol concentrations. Psychoneuroendocrinology, 37, 602-610.

Staufenbiel, S. M., Penninx, B. W. J. H., Spijker, A. T., Elzinga, B. M., \& van Rossum, E. F. C. (2013). Hair cortisol, stress exposure, and mental health in humans: A systematic review. Psychoneuroendocrinology, 38, 12201235.
Vanaelst, B., De Vriendt, T., Huybrechts, I., Rinaldi, S., De Henauw, S. (2012). Epidemiological approaches to measure childhood stress. Paediatric and Perinatal Epidemiology, 26, 280-297.

Van Os, J., \& Jones, P. B. (2001). Neuroticism as a risk factor for schizophrenia. Psychological Medicine, 31, 1129-1134.

Van Os, J., Rutten, B. P., \& Poulton, R. (2008). Geneenvironment interactions in schizophrenia: Review of epidemiological findings and future directions. Schizophrenia Bulletin, 34, 1066-1082.

Vliegenthart, J., Noppe, G., van Rossum, E. F. C., Koper, J. W., Raat, H., \& van den Akker, E. L. T. (2016). Socioeconomic status in children is associated with hair cortisol levels as a biological measure of chronic stress. Psychoneuroendocrinology, 65, 9-14.

Wells, S., Tremblay, P. F., Flynn, A., Russell, E., Kennedy, J., Rehm, J., \& Graham, K. (2014). Associations of hair cortisol concentration with self-reported measures of stress and mental health-related factors in a pooled database of diverse community samples. Stress, 17, 334-342.

Wilson, M.C., Zilioli, S., Ponzi, D., Henry, A., Kubicki, K., Nickels, N., \& Maestripieri, D. (2015).Cortisol reactivity to psychosocial stress mediates the relationship between extraversion and unrestricted sociosexuality. Personality and Individual Differences, 86, 427-431.

Wray, N. R., Coventry, W. L., James, M. R., Montgomery, G. W., Eaves, L. J., \& Martin, N. G. (2008). Use of monozygotic twins to investigate the relationship between 5HTTLPR genotype, depression and stressful life events: An application of Item Response Theory. Novartis Foundation Symposium, 293, 48-59; discussion 59-70.

Wright, M., \& Martin, N. G. (2004). Brisbane adolescent twin study: Outline of study methods and research projects. Australian Journal of Psychology, 56, 65-78. 